\title{
A Balance Principle Approach for Modeling Phase Transformation Kinetics
}

\author{
M. Lusk, G. Krauss* and H.-J. Jou
}

Division of Engineering, Colorado School of Mines, Golden, CO 80401, U.S.A.

* Department of Metallurgical and Materials Engineering, Colorado School of Mines, Golden, CO 80401, U.S.A.

\begin{abstract}
A balance principle is offered to model volume fraction kinetics of phase transformation kinetics at a continuum level. This microbalance provides a differential equation for transformation kinetics which is coupled to the differential equations governing the mechanical and thermal aspects of the process. Application here is restricted to diffusive transformations for the sake of clarity, although the principle is discussed for martensitic phase transitions as well. Avrami-type kinetics are shown to result from a special class of energy functions. An illustrative example using a $0.5 \% \mathrm{C}$ Chromium steel demonstrates how TTT and CCT curves can be generated using a particularly simple effective energy function.
\end{abstract}

\section{INTRODUCTION}

The kinetics of solid state phase transformations are dependent on the mechanical and thermal processes imposed on a material. This has motivated the development of a number of predictive models which couple thermomechanical state to phase evolution. At a length scale where individual phase interfaces are distinguishable, modeling efforts often track the evolution of a chemical species and/or a set of order parameters by postulating a kinetic equation that relates their rate of change to variations in the free energy with respect to that parameter. Landau-Ginzburg and Cahn-Hilliard kinetics are classical examples. Another approach is to track the motion of sharp phase interfaces with kinetic equations based on the conditions that are thought to exist on either side of such boundaries. In any case, however, problems of engineering interest often focus on a much larger length scale. It is difficult to develop kinetic relations at this level which take into account not only the phase interfaces, but also the other microstructural features that influence the transformation. Such efforts must make a number of idealizing assumptions which allow a volume fraction model to be built up from a consideration of the evolution of the nucleation and growth of stable nuclei. In diffusive transformations, for instance, a notable success of this kind is the Johnson-Mehl-AvramiKolmorgorov (JMAK) equation, valid under isothermal conditions:

$$
\phi(t)=1-e^{-k t^{n}}
$$

where $k$ and $n$ are quantities derived from a consideration of nucleation and growth rates combined with product phase geometry, and $\phi$ is the product volume fraction which varies with time $t$. If $n$ is not a function of temperature, then isothermal solutions can also be used to predict the volume fraction as a function time for a prescribed cooling history. This is known as the Rule of Additivity, and the approach has been popularized in a number of commercially available numerical codes [1]. For athermal martensite formation, a number of formulations akin to the Koistinen-Marburger (K-M) equation are developed along similar lines.

In both types of transformations, empirical fits are used to modify derived quantities such as $k$ and $n$ in order to make the kinetic relations of practical use. The end result is a combination of rigorous analysis and experimental curve fitting. In addition to the issue of adjusting physically derived parameters, it is not obvious how to generalize such models to account for mechanical effects and the competitive formation of multiple product microstructures. One thought is to try to develop a self-consistent kinetic model at the volume fraction level that addresses these deficiencies.

Underlying most kinetic models is the thermodynamic reasoning that the rate at which a transformation occurs is related to the thermodynamic driving force and energy barriers to phase growth. On the micromechanical scale, this idea has been formalized as a microforce balance principle in two foundational works 
by Fried and Gurtin [2,3]. They show how the Ginzburg-Landau and Cahn-Hilliard equations, along with a number of relaxation laws used in the past, can be derived from this single postulate. Fried and Gurtin also demonstrate that kinetic relations for the velocity of sharp interfaces $[4,5,6]$ are recovered in the asymptotic limit. Developing a volume fraction kinetics model from such a perspective is just as challenging as previous earlier efforts to derive an overall kinetic equation. Significantly, though, the microforce kinetic equation couples naturally to the heat and stress equations, and this suggests at least a framework for developing a more inclusive volume fraction kinetics model.

In the present work, a kinetic balance principle is used to model phase transformations in carbon steels based on the notion of an effective free energy and an effective mobility function. The length scale adopted is such that individual phases are identified by their volume fraction. The formal framework of Fried and Gurtin is used to model the formation of a single product phase without including mechanical effects. For diffusive transformations, the resulting kinetic equation can be solved for fixed temperatures to generate TTT diagrams. Numerical solutions can also be obtained under nonisothermal conditions, in full coupling with the (generalized) heat equation, without making an appeal to the Rule of Additivity. For martensitic phase transitions, the temperature dependence of a well of the effective energy function can be related to the K-M equation of martensite kinetics. Significantly, competitive formations can be studied with multiple, coupled kinetic equations, and mechanical effects can be brought in naturally. This logical framework is not derived from small scale kinetic principles and is intended to be a practical tool in performing quantitative analyses of engineering interest.

\section{BASIC FORMULATION}

Since the model being presented generalizes the purely thermal case, thermal processes without phase transformations will be briefly reviewed. Continuum models of temperature variation are based on the balance of energy along with a statement of the second law of thermodynamics. These postulates are expressed in terms of:

$\begin{array}{llll}R & \text { arbitrary piece of material } & \theta & \text { temperature } \\ \partial R & \text { the closed boundary of } R & \eta & \text { entropy } \\ \mathbf{n} & \text { outward unit normal vector to } \partial R & \mathbf{q} & \text { heat flux out of } R\end{array}$

$\varepsilon \quad$ internal energy

The energy and entropy postulates are, respectively:

$$
\frac{d}{d t} \int_{R} \varepsilon \mathrm{dV}=\int_{\partial R} \mathbf{q} \cdot \mathbf{n} \mathrm{dA}, \quad \frac{d}{d t} \int_{R} \eta \mathrm{dV} \geq \int_{\partial R} \frac{\mathbf{q} \cdot \mathbf{n}}{\theta} \mathrm{dA}
$$

where supply terms have been suppressed for the sake of clarity. The energy balance localizes to

$$
\dot{\varepsilon}=-\mathbf{u v}(\mathrm{q})
$$

where div is the divergence operator and a dot denotes differentiation with respect to time. This differential equation completely characterizes thermal processes provided the internal energy, entropy, and heat flux are given constitutive characterizations. The form of these descriptions is restricted by the second law of thermodynamics, with the simplest representation being that associated with isotropic constitutive functions having at most a linear dependence on the gradient of temperature. Under these conditions the energy balance becomes the heat equation:

$$
\hat{c}(\theta) \dot{\theta}=\operatorname{div}[\hat{k}(\theta) \nabla \theta]
$$

where $\hat{c}(\theta)$ is the specific heat and $\hat{k}(\theta)$ is the thermal diffusivity.

\subsection{Thermal processes with phase transformations}

Consistent with the approach of Fried and Gurtin for rationalizing a number of kinetic equations, we now suggest a logical framework for generalizing the purely thermal process to account for transformation kinetics based on volume fraction. Central to this approach is the addition of a microbalance principle used to describe the relationship between temperature and phase volume fraction during a phase transformation. Since the increase in one phase at the expense of another is called accretion, this balance principle is also referred to as an accretive force balance. For the sake of clarity, the mechanical aspect of the problem will be suppressed and only one product phase will be considered. We have also extended this approach to consider the competitive formation of multiple product phases.

The microbalance for phase volume fraction $\phi$ is given by

$$
\int_{\partial R} \xi \cdot \mathbf{n} \mathrm{dA}+\int_{R} \pi \mathrm{d} \mathbf{V}=0
$$


while the energy balance now includes a term associated with accretion:

$$
\frac{d}{d t} \int_{R} \varepsilon \mathrm{dV}=-\int_{\partial R} \mathbf{q} \cdot \mathbf{n} \mathrm{dA}+\int_{R} \dot{\phi} \xi \cdot \mathbf{n} \mathrm{dA}
$$

and the entropy imbalance is still given by equation $(2)_{2}$. Here $\xi$ is called the micro stress which expends power in proportion to the rate of change of volume fraction. $\pi$ is the micro internal force and does not expend power as it represents interactions on a smaller scale than that associated with the model. The micro and energy balances localize to:

$$
\operatorname{div}(\xi)+\pi=0, \quad \dot{\varepsilon}-\xi \cdot \nabla \dot{\phi}-\pi \dot{\phi}=-\operatorname{div}(\mathbf{q})
$$

In the absence of mechanical influences, it is these two differential equations that govern the phase transformation process. In order to use them, constitutive characterizations must first be given for the internal energy, entropy, heat flux, micro stress, and micro internal force. A reasonable assumption is that these fields depend on the temperature and its gradient and also product volume fraction, its gradient and its rate of change. If attention is restricted to isotropic materials and the assumption is made that the micro stress, micro internal force and heat flux have at most a linear dependence on the gradient of temperature, the rate of change of temperature, and the gradient of the volume fraction, then the second law of thermodynamics implies that the energy balance and the accretive force balance must be of the form:

$$
\begin{aligned}
\hat{c}(\theta, \phi) \dot{\theta} & =\operatorname{div}[\hat{k}(\theta, \phi) \nabla \theta]+\theta \hat{\psi}_{\theta \phi}(\theta, \phi, \nabla \phi) \dot{\phi}+\beta(\theta, \phi) \dot{\phi}^{2} \\
\beta(\theta, \phi) \dot{\phi} & =\lambda \Delta \phi-\hat{\psi}_{\phi}(\theta, \phi, \nabla \phi)
\end{aligned}
$$

where $\triangle$ is the Laplacian operator, a subscript implies partial differentiation, and:

$\begin{array}{llll}\hat{c}(\theta, \phi) & \text { specific heat } & \lambda & \text { constitutive constant } \\ \hat{k}(\theta, \phi) & \text { thermal diffusivity } & \hat{\psi}(\theta, \phi, \nabla \phi) & \text { Helmholtz free energy with } \psi=\varepsilon-\theta \eta \\ \hat{\beta}(\theta, \phi) & \text { mobility coefficient } & & \end{array}$

Note that $\lambda$ in the kinetic equation need not actually be a constant. Since no distinction has been made at this point between diffusive and martensitic transitions, these two differential equations can be compared with those associated with both the JMAK model of diffusion and the K-M model of martensite formation.

The Helmholtz free energy of the material is allowed to depend on the gradient of the product volume fraction. This term accounts for the fact that the specific energy is higher in regions where there is a spatially sharp transition between the parent and product phases. Significantly, this dependence on $\nabla \phi$ does not differentiate between two states with the same product volume fraction but different phase distributions at a smaller length scale. However, the distribution of phases has significant influence on the homogenized properties of the aggregate as do the interfaces associated with a given distribution. This explains why the energy function dependence on volume fraction is not simply a volume-weighted average.

\subsection{A simplified model for thermally driven phase transitions}

For phase transformations that are thermally induced, it is reasonable to expect that the spatial variation in phase volume fraction is negligibly smali provided the rate of cooling is sufficiently slow. Under such conditions, the micro stress vanishes and the governing equations can be written as

$$
\begin{aligned}
\hat{c}(\theta, \phi) \dot{\theta} & =\operatorname{div}[\hat{k}(\theta, \phi) \nabla \theta]+\hat{L}(\theta, \phi) \dot{\phi} \\
\beta(\theta, \phi) \dot{\phi} & =-\hat{\psi}_{\phi}(\theta, \phi)
\end{aligned}
$$

where $L$ is the latent heat, or enthalpy of transformation, given by

$$
\hat{L}(\theta, \phi)=-\hat{\psi}_{\phi}(\theta, \phi)+\theta \hat{\psi}_{\phi \theta}(\theta, \phi)=-\hat{\varepsilon}_{\phi}(\theta, \phi)
$$

This yields the appropriate interpretation of the enthalpy of transformation as the energy released when parent material is transformed to a product phase with lower internal energy. The term provides coupling between the thermal and accretive equations.

If the component is sufficiently small and/or the cooling rate sufficiently slow, then the heat generation due to the enthalpy of transformation may be negligible. In such cases the cooling rate can be prescribed as a boundary condition and approximated as constant throughout the specimen. The governing equations then reduce to:

$$
\dot{\theta}=\hat{\Theta}(t), \quad \dot{\phi}=\frac{-1}{\hat{\beta}(\theta, \phi)} \hat{\psi}_{\phi}(\theta, \phi)
$$


where $\hat{\Theta}(t)$ is the prescribed cooling history of the part. This gives a simple setting in which to compare the accretive force balance with more standard approaches for modeling phase transformation kinetics. In this initial study, no attempt is made to separate out the effects of the mobility coefficient, $\beta$, and the derivative of the free energy, $\psi_{\phi}$. The mobility coefficient is therefore absorbed by the energy function in what follows.

\subsection{Examples of Energy Functions}

To demonstrate the relationship between the JMAK approach and that of the microbalance, equation (1) can be recast in the differential form

$$
\dot{\phi}=-\hat{\psi}_{\phi}(\theta, \phi)=n k^{\frac{1}{n}}(1-\phi)[-\operatorname{In}(1-\phi)]^{\frac{n-1}{n}}
$$

This gives the derivative of the energy function which can then be integrated. The resulting energy for JMAK kinetics is shown in Figure 1(a). As is clear from equation (12), the rate at which a transformation proceeds is proportional to the derivative of the free energy with respect to volume fraction. A phase transformation occurs when the current state is no longer a local minimum. The energy function associated with JMAK kinetics has, for all temperatures, a maximum for a product volume fraction of zero and a minimum for a volume fraction of one. This implies that the product phase is preferred for all temperatures and that the reaction always goes to completion.

Significantly, equation (12) can be solved for any cooling history, $\Theta(t)$, provided the temperature dependence of $n$ and $k$ is known. Such solutions will be the same as those obtained using the Rule of Additivity provided $n$ is not a function of temperature-a necessary condition for the Rule of Additivity to be valid [1].

For comparative purposes, Figure 1(b) shows an alternate energy function for phase transition kinetics:

$$
\psi=\phi^{k}\left(\frac{\phi^{3}}{3+\phi}-\frac{\phi^{2}(1-n)}{2+k}-\frac{\phi n}{1+k}\right)
$$

where $n$ and $k$ are functions of temperature. This function has a more standard two-well system with the relative well height a function of temperature. Absorption of the kinetic coefficient $\beta$ into the parameter $k$ gives the following kinetic equation for this energy function:

$$
\dot{\phi}=\phi^{k}(1-\phi)(n+\phi)
$$

where again, $k$ and $n$ can be functions of temperature. It can be shown that this kinetic equation is capable of modeling experimentally obtained TTT data, but an even simpler model will be used in the next section to demonstrate a practical application.
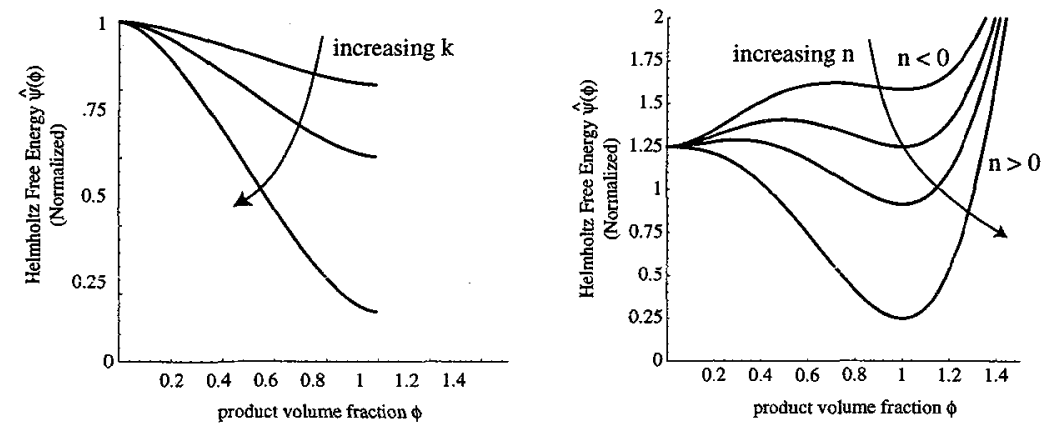

Figure 1: (a) The free energy function associated with JMAK kinetics; (b) an alternate energy free energy function with $n$ varying and $k$ fixed.

\section{Practical Example: Generation of TTT and CCT Curves}

As an illustration of the practical application of this theory, the pearlite component of TTT and CCT curves will be generated for a $0.5 \% \mathrm{C}$ Chromium steel using a particularly simple energy function. Let the energy be given by

$$
\hat{\psi}(\theta, \phi)=-\hat{\nu}(\theta) \phi^{2}\left(\frac{1}{2}-\frac{\phi}{3}\right)
$$

so that the kinetics are governed by

$$
\dot{\phi}=\nu \phi(1-\phi), \quad \phi(0)=\phi_{0}
$$

where, again, the mobility coefficient $\beta$ has been absorbed into the energy function for the sake of simplicity. Both $\nu$ and the initial product volume fraction $\phi_{o}$ must be determined empirically. The first application 
used the commercially available Minitech [7] software to make predictions of the isothermal kinetics with a subsequent use of separate curve fitting software to obtain a functional representation for the required parameters. In practice, experimentally obtained data would be used instead. Figure 2 shows the energy to within an arbitrary constant for the alloy chosen along with the function fit obtained for the energy parameter $\nu$. The initial volume fraction was determined to be a constant $\phi_{o}=.00138$.
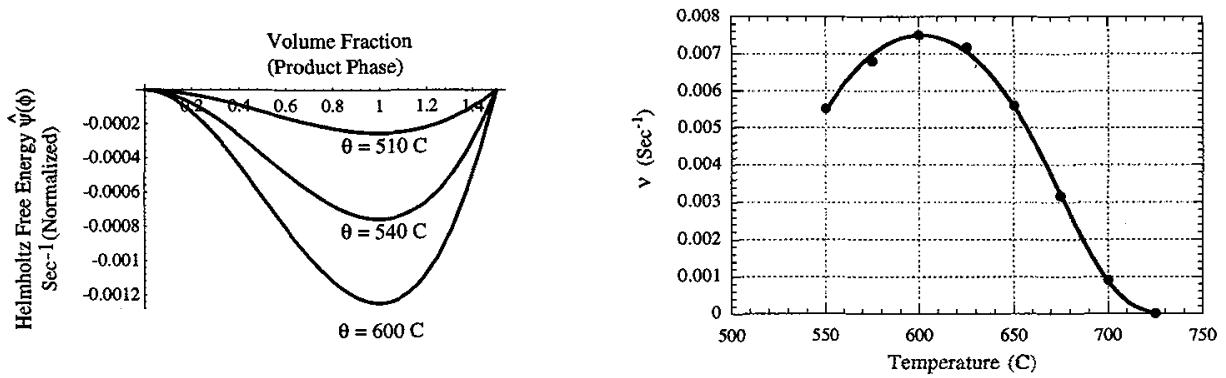

Figure 2: (a) The energy potential for a $0.5 \% \mathrm{C}$ Chromium steel; (b) the associated kinetic parameter $\hat{\nu}(\theta)$.

This information was applied to the kinetic relation of equation (16) in a Fortran code to obtain the $1 \%$ and $99 \%$ TTT curves shown in Figure 3 (a) along with the Minitech data. The results indicate that the simple energy function chosen can accurately reproduce TTT curves. The same approach was then used with atlas data [8] for the same steel, and the results are shown in Figure 3(b). It should be noted that the atlas provided only two data points at each temperature with which to obtain parameter fits. Here a spline fit was generated for the function $\hat{\nu}(\theta)$ and the differential equation solver was generalized to handle spline functions.
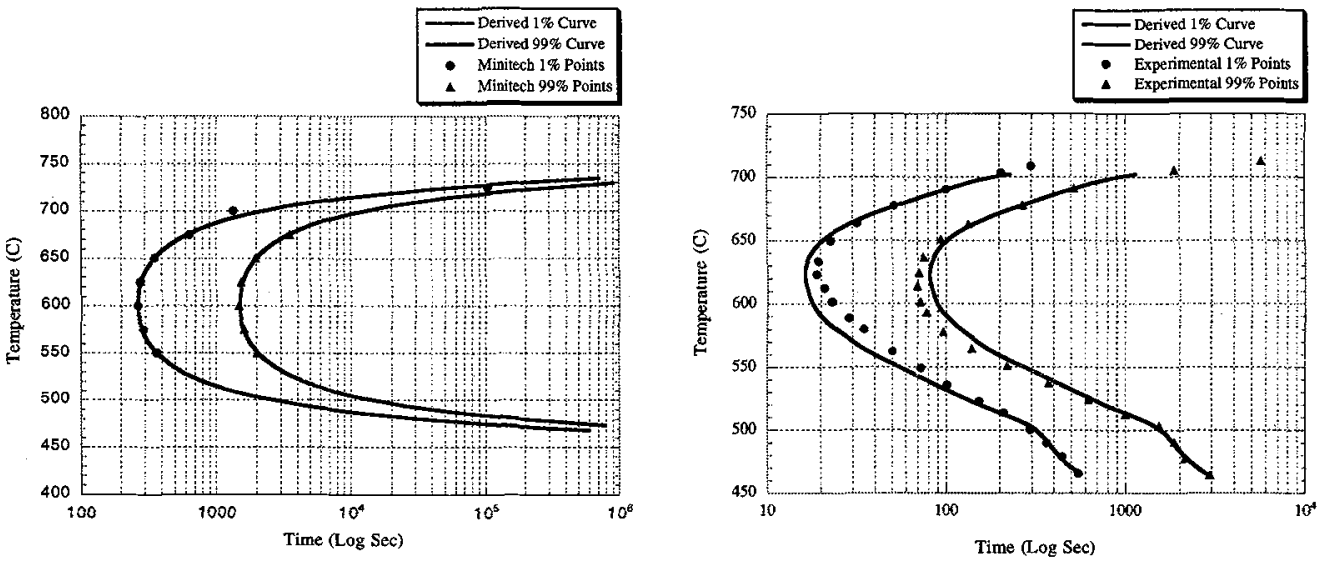

Figure 3: Pearlite TTT curves for a $0.5 \% \mathrm{C}$ Chromium steel. (a) derived curves and Minitech data points; (b) experimentally obtained curves.

Once the temperature dependence is established for the coefficient $\nu$ the kinetic equation can be solved numerically for any cooling history. For example, Figure 4(a) shows the results obtained for exponential cooling based on the isothermal data from Minitech. These results are the same as those obtained using the Rule of Additivity since it is valid to apply the rule to this energy function [1]. Figure 4(b) compares atlas CCT curves with the CCT curves derived from the kinetic equation and isothermal atlas data. Although the derived curves are in reasonable agreement with experiment, we believe that a better agreement can be obtained if more than two data points were available for each isothermal test. 

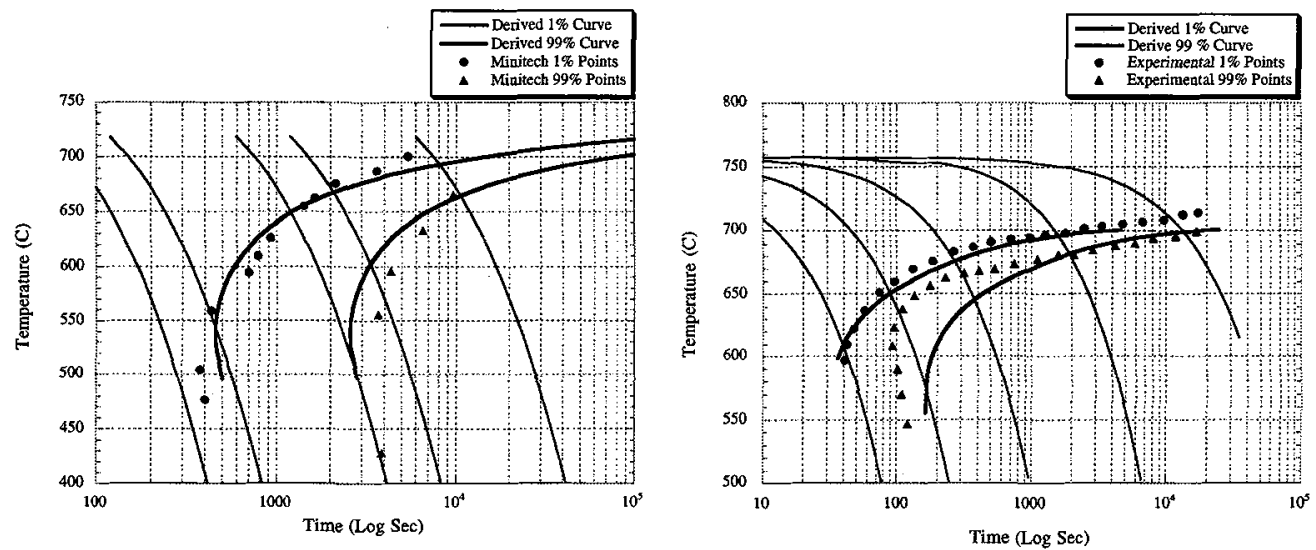

Figure 4: Pearlite CCT curves for a $0.5 \%$ C Chromium steel. (a) derived curves and Minitech data points; (b) experimentally obtained curves.

\section{Conclusions}

A microbalance principle has been used to model phase transformation kinetics at a volume fraction level, and it is argued that the approach can be applied to martensitic as well as diffusive processes. The energy dependence on volume fraction is intended to capture small scale homogenization effects as well as interface effects and leads to a natural definition of the enthalpy of transformation. A special class of energy functions yield JMAK kinetics and a particularly simple energy function was applied to a $0.5 \% \mathrm{C}$ Chromium steel to generate both TTT and CCT curves. In addition to the improvements anticipated with more complete constitutive data, more sophisticated energy functions and a consideration of multiple product phases should also result in more accurate predictions of nonisothermal material behavior.

In the analysis of sections 2 and 3 , the cooling history was prescribed and so no consideration was given to the form of the specific heat or enthalpy of formation. However, both of these quantities can be derived from the single energy function by virtue of equation (10) and

$$
\hat{c}(\theta, \phi)=\hat{\varepsilon}_{\theta}(\theta, \phi)=-\hat{\psi}_{\theta \theta}(\theta, \phi)
$$

Moreover, no consideration was given to the form of the mobility coefficient $\hat{\beta}(\theta, \phi)$ and this is not derivable from the energy function. We have developed constitutive models which take the considerations into account and yield physically reasonable functions for the specific heat, the enthalpy of transformation, and the kinetic coefficient.

\section{Acknowledgments}

The first author gratefully acknowledges a number of helpful discussions with Eliot Fried and Morton Gurtin. This research has been supported, in part, by the National Center for Manufacturing Sciences through contract 02-14-0302-95005.

\section{References}

[1] Lusk, M. and H. Jou, submitted to Acta Met. (1995).

[2] Fried, E. and Gurtin, M., Phys. D 72 (1994) 287-308.

[3] Fried, E. and Gurtin, M., Phys. D 68 (1993) 326-343.

[4] Gurtin, M., Arch. Ration. Mech. Analysis 104 (1988) 195-221.

[5] Angenent, S. and Gurtin, M., Arch. Ration. Mech. Analysis 108 (1989) 323-391.

[6] Gurtin, M. and Struthers, A., Arch. Ration. Mech. Analysis 112 (1990) 97-160.

[7] The Minitech Computerized Alloy Steel Information System, Minitech Limtd., Hamilton, Ontario.

[8] Vander Voort, G. (Ed.), Atlas of Time-Temperature Diagrams for Irons and Steels, (ASM International, 1991) p. 134. 